Supporting Information for

\title{
Electrochemical synthesis of $\beta$-ketosulfones from switchable starting materials
}

\author{
Issa Yavari*, Sina Shaabanzadeh
}

Department of Chemistry, Tarbiat Modares University, P.O. Box 14115-175, Tehran, Iran

Table of Contents

\begin{tabular}{|ll|}
\hline I. General Information & S2 \\
\hline II. Cyclic voltammograms & S3-S7 \\
\hline III. Experimental Section & S8 \\
\hline IV. Spectral data & S9-S12 \\
\hline V. Copies of ${ }^{1} \mathrm{H}$ NMR, Mass and IR & S13-S20 \\
\hline VI. Reference & S21 \\
\hline
\end{tabular}




\section{I-General Information}

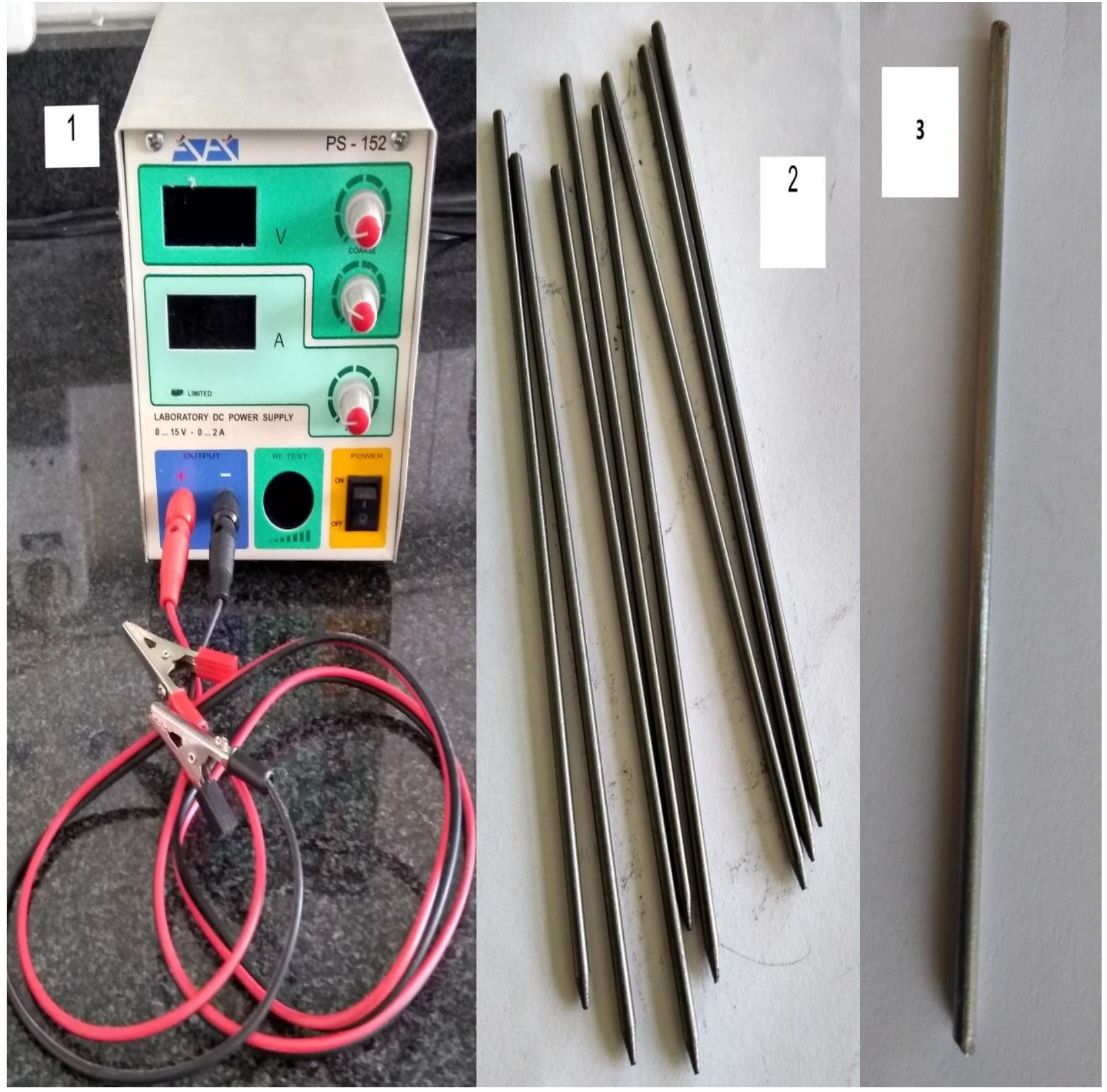

Figures. (1) The picture of DC power supply (2) pencil graphite electrode (3) stainless steel electrode. 


\section{II- Cyclic Voltammograms}

To clarify the role of substrates present in these transformations, cyclic voltammetry (CV) experiments were performed. As shown in Figures 4-9, the analyses were recorded at $-2 \mathrm{~V}$ to $2 \mathrm{~V}$ potential range with unreactive $\mathrm{LiClO}_{4}$ as an electrolyte. In $\mathrm{MeOH}$ cyclic voltammetry cathodic reduction curve in Figure 4 showed a peak at $-1.08 \mathrm{~V}$ (vs. $\mathrm{Ag} / \mathrm{AgCl}$ ) that was attributed to the formation of $\mathrm{H}_{2}$ and methoxide ion $\left(\mathrm{MeO}^{-}\right)$. By analyzing acetophenone $\mathrm{CV}$ in $\mathrm{MeOH}$ reduction peaks of acetophenone were observed upper than 0.6 and $-1.8 \mathrm{~V}(v s . \mathrm{Ag} / \mathrm{AgCl})$ as reported previously (Figure 5). The anodic oxidation peaks of potassium iodide were showed at 0.39 and $0.57 \mathrm{~V}(v s . \mathrm{Ag} / \mathrm{AgCl})$, respectively and the

$1.95 \mathrm{~V}(v s . \mathrm{Ag} / \mathrm{AgCl})$ oxidation peak maybe related to oxidation $\mathrm{MeOH}$ in presence of iodide (Figure 6). For ensuring about the $1.95 \mathrm{~V}(v s . \mathrm{Ag} / \mathrm{AgCl}$ ) peak we repeated $\mathrm{KI}$ iodide cyclic voltammetry in absolute ethanol because of KI sparingly soluble at room temperature but solubility can be increased by warming up. The results showed that the $1.95 \mathrm{~V}(v s . \mathrm{Ag} / \mathrm{AgCl}$ ) peak probably belongs to oxidation alcohol in presence KI (Figure 7). The CV of phenylacetylene did not show any clear oxidation or reduction peak, and it apparently remains intact under electrochemical condition (Figure 8). As showed in Figure 9 and 10 sodium sulfinate salts have three oxidation peak near $0.8,1.4$ and $1.8 \mathrm{~V}$ ( $v s$. $\mathrm{Ag} / \mathrm{AgCl}$ ) respectively. ${ }^{5}$ 


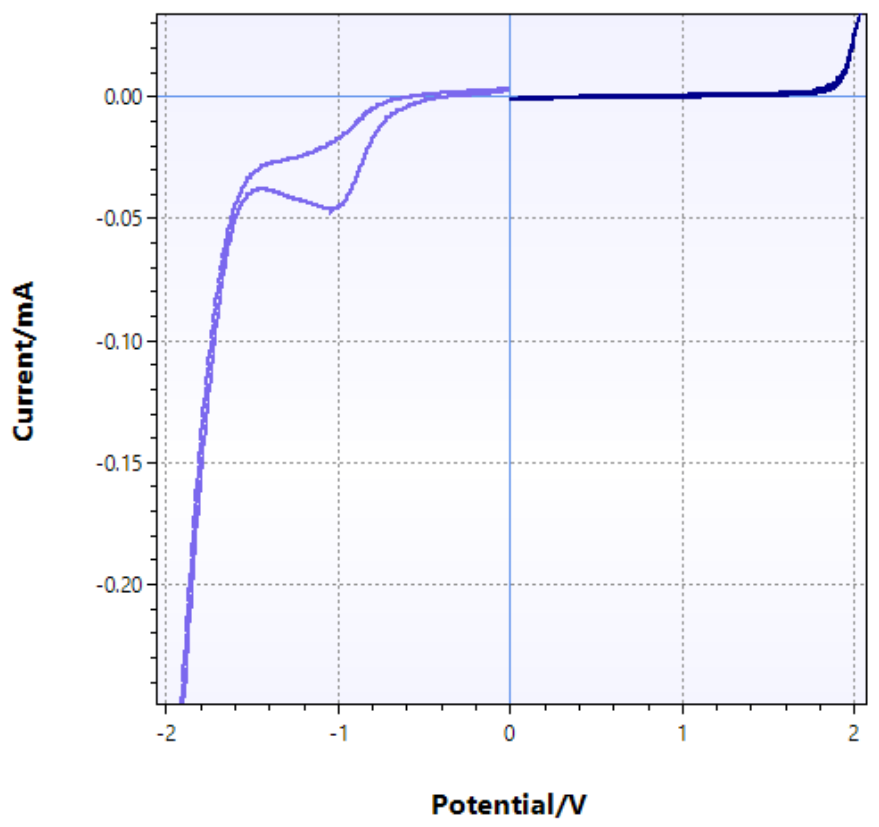

Figure 4 cyclic votammogram of $10 \mathrm{~mL} \mathrm{MeOH}$ in the presence of $\mathrm{LiClO}_{4}(0.1 \mathrm{M})$ recorded at a glassy carbon electrode $(2.0 \mathrm{~mm}$ diameter $)$, sweep rate: $0.1 \mathrm{~V} / \mathrm{s}$, at $\mathrm{rt}$, $\mathrm{Ag} / \mathrm{AgCl}$ as a reference electrode.

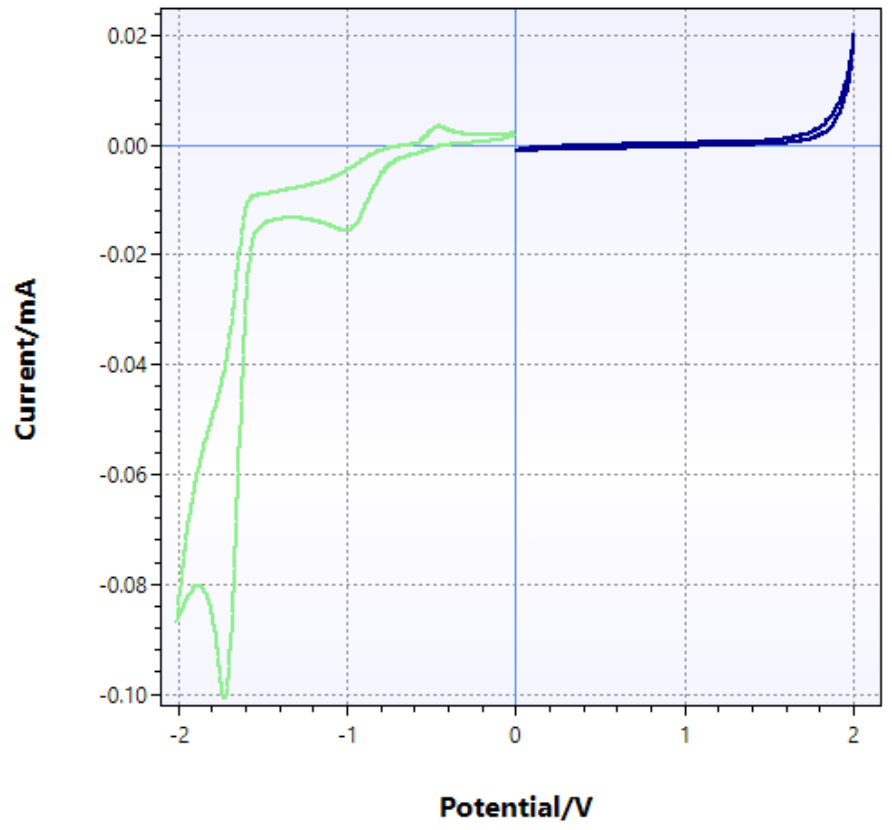

Figure 5 cyclic votammogram of $1 \mathrm{mmol}$ of $\mathbf{1 a}$ in $10 \mathrm{~mL} \mathrm{MeOH}$ in the presence of $\mathrm{LiClO}_{4}(0.1 \mathrm{M})$ recorded at a glassy carbon electrode $(2.0 \mathrm{~mm}$ diameter $)$, sweep rate: 0.1 $\mathrm{V} / \mathrm{s}$, at $\mathrm{rt}, \mathrm{Ag} / \mathrm{AgCl}$ as a reference electrode. 


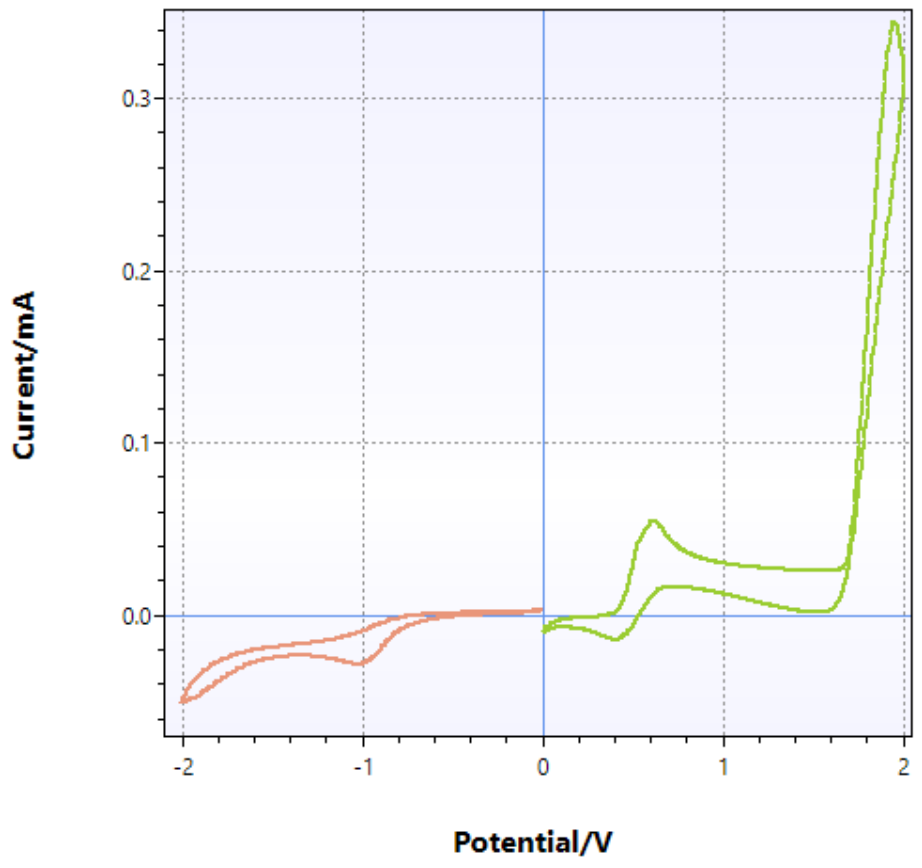

Figure 6 Cyclic votammogram of $\mathrm{MeOH}(10 \mathrm{~mL})$ in the presence of $\mathrm{LiClO}_{4}(0.1 \mathrm{M})$ in the presence of $\mathrm{KI}(0.1 \mathrm{M})$ recorded at a glassy carbon electrode $(2.0 \mathrm{~mm}$ diameter), sweep rate: $0.1 \mathrm{~V} / \mathrm{s}$, at $\mathrm{rt}, \mathrm{Ag} / \mathrm{AgCl}$ as a reference electrode.

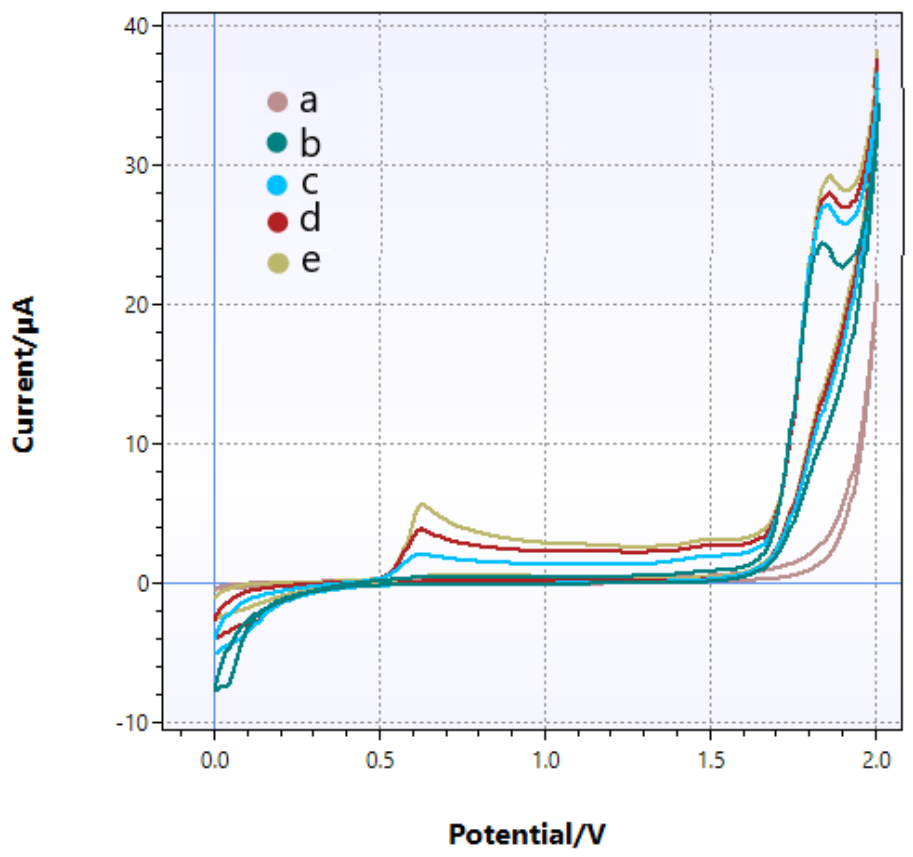

Figure 7 Cyclic votammogram of $\mathrm{EtOH}(10 \mathrm{~mL})$ in the presence of $\mathrm{LiClO}_{4}(0.1 \mathrm{M})$ in the a) absence of $\mathrm{KI}$ at rt, b) presence of $\mathrm{KI}(166 \mathrm{mg})$ at rt, c) presence of $\mathrm{KI}(166 \mathrm{mg})$ at $40{ }^{\circ} \mathrm{C}$, c) presence of $\mathrm{KI}(166 \mathrm{mg})$ at $50{ }^{\circ} \mathrm{C}$, c) presence of $\mathrm{KI}(166 \mathrm{mg})$ at $60{ }^{\circ} \mathrm{C}$ recorded at a glassy carbon electrode ( $2.0 \mathrm{~mm}$ diameter), sweep rate: $0.1 \mathrm{~V} / \mathrm{s}, \mathrm{Ag} / \mathrm{AgCl}$ as a reference electrode. 


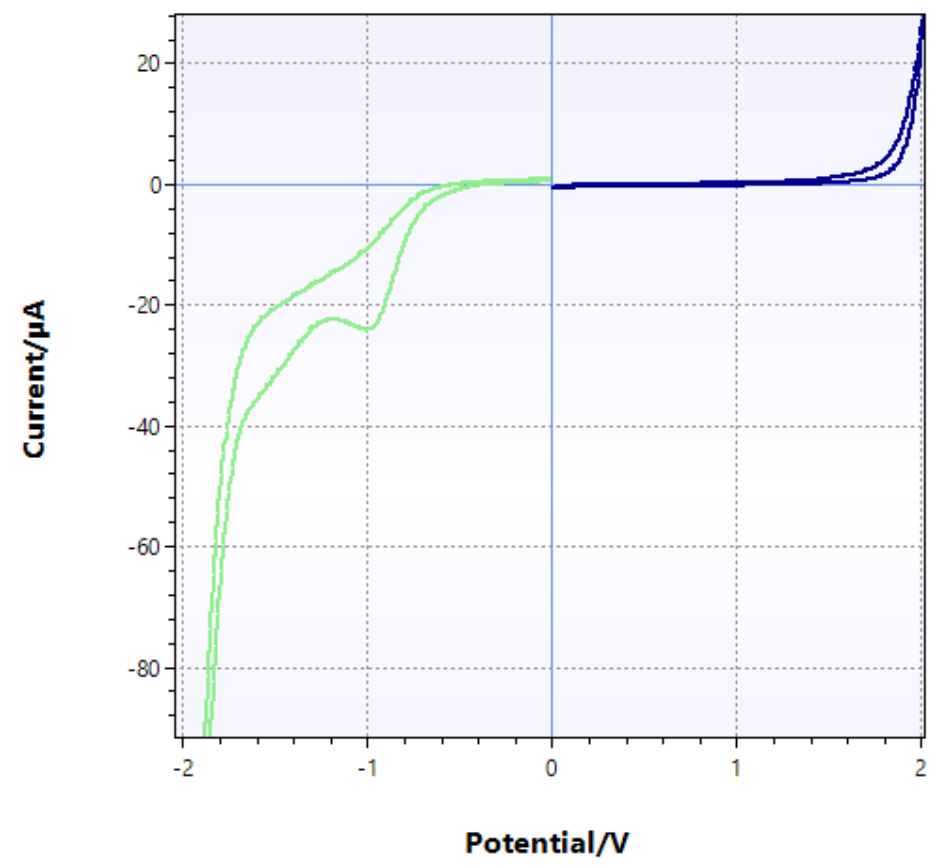

Figure 8 cyclic votammogram of $1 \mathrm{mmol}$ of $\mathbf{4 a}$ in $10 \mathrm{~mL} \mathrm{MeOH}$ in the presence of $\mathrm{LiClO}_{4}(0.1$ $\mathrm{M})$ recorded at a glassy carbon electrode $(2.0 \mathrm{~mm}$ diameter $)$, sweep rate: $0.1 \mathrm{~V} / \mathrm{s}$, at rt, $\mathrm{Ag} / \mathrm{AgCl}$ as a reference electrode.

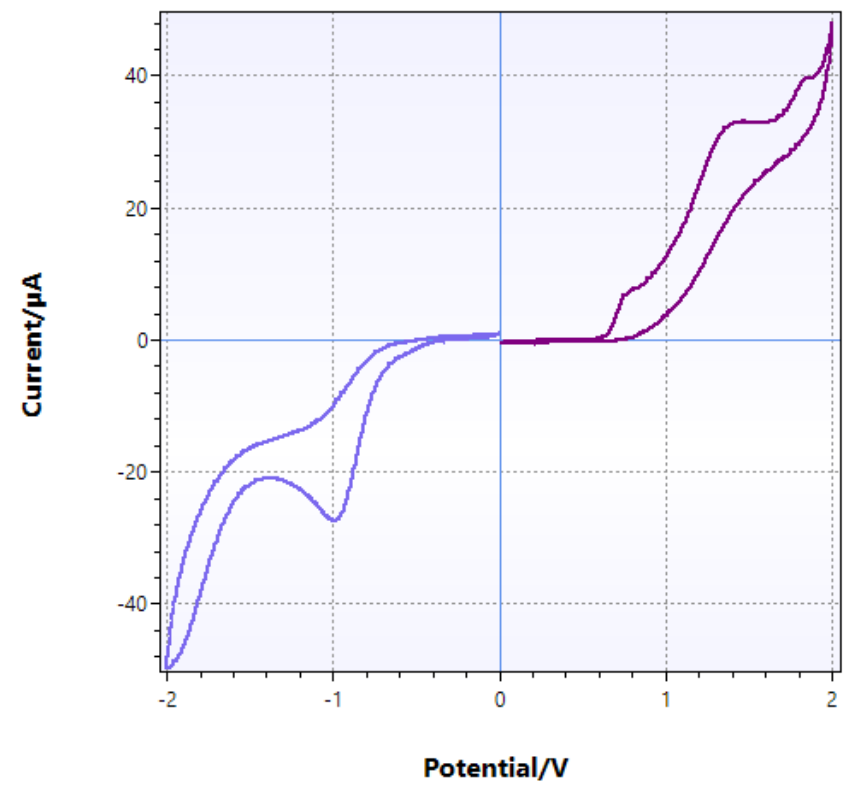

Figure 9 cyclic votammogram of $1 \mathrm{mmol}$ of $\mathrm{PhSO}_{2} \mathrm{Na}$ in $10 \mathrm{~mL} \mathrm{MeOH}$ in the presence of $\mathrm{LiClO}_{4}(0.1 \mathrm{M})$ recorded at a glassy carbon electrode $(2.0 \mathrm{~mm}$ diameter $)$, sweep rate: $0.1 \mathrm{~V} / \mathrm{s}$, at $\mathrm{rt}, \mathrm{Ag} / \mathrm{AgCl}$ as a reference electrode. 


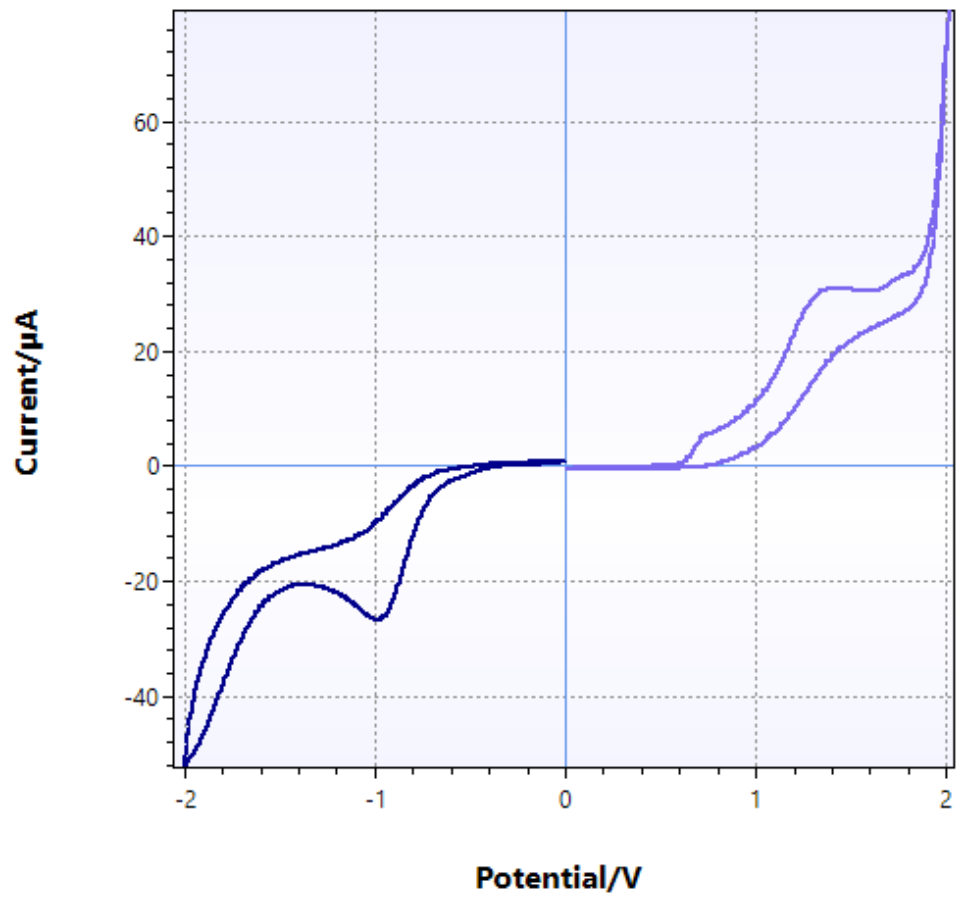

Figure 10 cyclic votammogram of $1 \mathrm{mmol}$ of $\mathrm{TolSO}_{2} \mathrm{Na}$ in $10 \mathrm{~mL} \mathrm{MeOH}$ in the presence of $\mathrm{LiClO}_{4}(0.1 \mathrm{M})$ recorded at a glassy carbon electrode $(2.0 \mathrm{~mm}$ diameter), sweep rate: $0.1 \mathrm{~V} / \mathrm{s}$, at $\mathrm{rt}, \mathrm{Ag} / \mathrm{AgCl}$ as a reference electrode. 


\section{III-Experimental Section}

All purchased solvents and chemicals were of analytical grade and used without further purification. Pencil graphite electrodes were $2.0 \mathrm{~mm} 2 \mathrm{~B}$ and purchased from the Stationery stores. Melting points and IR spectra of all compounds were measured on an Electrothermal 9100 apparatus and a Shimadzu IR-460 spectrometer, respectively. The 1H NMR spectra were obtained with a BRUKER DRX-500 AVANCE instrument using CDCl3 as applied solvent at $500 \mathrm{MHz}$. Mass spectra were recorded on a FINNIGAN-MAT 8430 mass spectrometer operating at an ionization potential of $70 \mathrm{eV}$.

\section{A typical procedure for the preparation of 3 a from $1 \mathrm{a}$}

A mixture of $\mathbf{1 a}(60 \mathrm{mg}, 0.5 \mathrm{mmol}), \mathbf{2 a}(178 \mathrm{mg}, 1 \mathrm{mmol})$ and $\mathrm{KI}(207.5 \mathrm{mg}, 0.25 \mathrm{M})$ in $\mathrm{MeOH}$ $(5 \mathrm{~mL})$ was stirred at rt for 15 minutes. An undivided cell was equipped with $\mathrm{St}$ (stainless steel) as cathode and PGE as anode (120 mm height and $2.0 \mathrm{~mm}$ diameter, 2B) connected with a DC power supply, the reaction was allowed to stir and the electrolysis was carried out at a constant current of $40 \mathrm{~mA}\left(16.6 \mathrm{~mA} / \mathrm{cm}^{2}\right)$ at $\mathrm{rt}$ for 5 hours until the quantity of the electricity $14.928 \mathrm{~F} / \mathrm{mol}$ was passed. After completion of the reaction (the progress of the reaction was followed by TLC), the solvent was removed under reduced pressure, and the residue was purified by column chromatography (silica gel 230-400 mesh; Merck, n-hexane/AcOEt 5:1) to give the desired product.

\section{A typical procedure for the preparation of 5 a from $4 a$}

A mixture of $\mathbf{4 a}(51 \mathrm{mg}, 0.5 \mathrm{mmol}), \mathbf{2 a}(178 \mathrm{mg}, 1 \mathrm{mmol}), \mathrm{KI}(207.5 \mathrm{mg}, 0.25 \mathrm{M})$ and TBHP $70 \%$ in $\mathrm{H}_{2} \mathrm{O}$ (64.25 mg, 2 equiv) in $\mathrm{MeOH}(5 \mathrm{~mL})$ was stirred at $\mathrm{rt}$ for 15 minutes. An undivided cell was equipped with St (stainless steel) as cathode and PGE as anode (120 mm height and $2.0 \mathrm{~mm}$ diameter, 2B) connected with a DC power supply, the reaction was allowed to stir and the electrolysis was carried out at a constant current of $40 \mathrm{~mA}\left(16.6 \mathrm{~mA} / \mathrm{cm}^{2}\right)$ at $\mathrm{rt}$ for 10 hours until the quantity of the electricity $29.857 \mathrm{~F} / \mathrm{mol}$ was passed. After completion of the reaction (the progress of the reaction was followed by TLC), the solvent was removed under reduced pressure, and the residue was purified by column chromatography (silica gel 230-400 mesh; Merck, $n$ hexane/AcOEt 5:1) to give the desired product. 


\section{III- Spectral data}

\section{1-Phenyl-2-tosylethanone (3a)}

Colorless solid, (110 mg) 82\% yield, mp: $105-107^{\circ} \mathrm{C}$. IR (KBr): 1142, $1315(\mathrm{~S}=\mathrm{O}), 1686(\mathrm{C}=\mathrm{O})$ $\mathrm{cm}^{-1} .{ }^{1} \mathrm{H}$ NMR $\left(500 \mathrm{MHz}, \mathrm{CDCl}_{3}\right) \delta 7.97(\mathrm{~d}, J=7.7 \mathrm{~Hz}, 2 \mathrm{H}), 7.79(\mathrm{~d}, J=8.0 \mathrm{~Hz}, 2 \mathrm{H}), 7.65(\mathrm{t}, J$ $=7.5 \mathrm{~Hz}, 1 \mathrm{H}), 7.51(\mathrm{t}, J=7.7 \mathrm{~Hz}, 2 \mathrm{H}), 7.36(\mathrm{~d}, J=7.9 \mathrm{~Hz}, 2 \mathrm{H}), 4.74(\mathrm{~s}, 2 \mathrm{H}), 2.47(\mathrm{~s}, 3 \mathrm{H})$.

Known compound. ${ }^{1}$<smiles>O=C(CS(=O)(=O)[GeH3])c1ccccc1</smiles>

\section{1-(o-Tolyl)-2-tosylethan-1-one (3b)}

Colorless solid, (115 mg) 80\% yield, mp: $110-112{ }^{\circ} \mathrm{C} .{ }^{1} \mathrm{H}$ NMR $\left(500 \mathrm{MHz}, \mathrm{CDCl}_{3}\right) \delta 7.81-7.73$ (m, 3H), $7.45(\mathrm{t}, J=7.6 \mathrm{~Hz}, 1 \mathrm{H}), 7.35(\mathrm{~d}, J=7.9 \mathrm{~Hz}, 2 \mathrm{H}), 7.33-7.28(\mathrm{~m}, 2 \mathrm{H}), 4.71(\mathrm{~s}, 2 \mathrm{H})$, 2.47 (s, 3H), 2.46 (s, 3H). Known compound. ${ }^{1}$<smiles>Cc1ccccc1C(=O)CS(=O)(=O)[AlH2]</smiles>

\section{1-(2-Chlorophenyl)-2-tosylethanone (3c)}

Colorless solid, $(130 \mathrm{mg}) 86 \%$ yield, mp: $95-97{ }^{\circ} \mathrm{C} .{ }^{1} \mathrm{H}$ NMR $\left(500 \mathrm{MHz}, \mathrm{CDCl}_{3}\right) \delta 7.79(\mathrm{~d}, J=$ $7.9 \mathrm{~Hz}, 2 \mathrm{H}), 7.58(\mathrm{~d}, J=7.7 \mathrm{~Hz}, 1 \mathrm{H}), 7.49-7.33(\mathrm{~m}, 5 \mathrm{H}), 4.84$ (s, 2H), 2.47 (s, 3H). Known compound. ${ }^{2}$<smiles>O=C(CS(=O)(=O)[AlH2])c1ccccc1Cl</smiles>

\section{1-(2-Methoxyphenyl)-2-tosylethanone (3d)}

Colorless solid, (115 mg) 77\% yield, mp: 84-85 ${ }^{\circ} \mathrm{C} .{ }^{1} \mathrm{H}$ NMR $\left(500 \mathrm{MHz}, \mathrm{CDCl}_{3}\right) \delta 7.77(\mathrm{~d}, J=$ $7.9 \mathrm{~Hz}, 2 \mathrm{H}), 7.67(\mathrm{t}, J=9.3 \mathrm{~Hz}, 1 \mathrm{H}), 7.52(\mathrm{t}, J=7.9 \mathrm{~Hz}, 1 \mathrm{H}), 7.35-7.26(\mathrm{~m}, 2 \mathrm{H}), 7.01(\mathrm{t}, J=$ 
$7.6 \mathrm{~Hz}, 1 \mathrm{H}), 6.92(\mathrm{~d}, J=8.4 \mathrm{~Hz}, 1 \mathrm{H}), 4.95(\mathrm{~s}, 2 \mathrm{H}), 3.91(\mathrm{~s}, 3 \mathrm{H}), 2.45$ (s, 3H). Known compound. $^{2}$<smiles>COc1ccccc1C(=O)CS(=O)(=O)Cl</smiles>

\section{1-(4-Fluorophenyl)-2-tosylethanone (3e)}

Colorless solid, (130 mg) 90\% yield, mp: $128-130{ }^{\circ} \mathrm{C} .{ }^{1} \mathrm{H}$ NMR $\left(500 \mathrm{MHz}, \mathrm{CDCl}_{3}\right) \delta 8.03(\mathrm{dd}, J$ $=8.5,5.3 \mathrm{~Hz}, 2 \mathrm{H}), 7.77(\mathrm{~d}, J=7.9 \mathrm{~Hz}, 2 \mathrm{H}), 7.37(\mathrm{~d}, J=8.6 \mathrm{~Hz}, 2 \mathrm{H}), 7.19(\mathrm{t}, J=8.5 \mathrm{~Hz}, 2 \mathrm{H})$, $4.71(\mathrm{~s}, 2 \mathrm{H}), 2.48$ (s, 3H). Known compound. ${ }^{2}$<smiles>O=C(CS(=O)(=O)Cl)c1ccc(F)cc1</smiles>

\section{1-(4-Bromophenyl)-2-tosylethanone (3f)}

Pale yellow solid, (152.5 mg) 88\% yield, mp: $141-144{ }^{\circ} \mathrm{C} .{ }^{1} \mathrm{H}$ NMR $\left(500 \mathrm{MHz}, \mathrm{CDCl}_{3}\right) \delta 7.90$ $(\mathrm{d}, J=7.8 \mathrm{~Hz}, 2 \mathrm{H}), 7.85(\mathrm{~d}, J=7.5 \mathrm{~Hz}, 2 \mathrm{H}), 7.71(\mathrm{t}, J=7.8 \mathrm{~Hz}, 1 \mathrm{H}), 7.66(\mathrm{dd}, J=8.4,1.8 \mathrm{~Hz}$, 2H), $7.59(\mathrm{t}, J=7.6 \mathrm{~Hz}, 2 \mathrm{H}), 4.72$ (s, 2H). Known compound. ${ }^{1}$<smiles>O=C(CS(=O)(=O)Cl)c1ccc(Br)cc1</smiles>

\section{1-(4-Iodophenyl)-2-tosylethanone (3g)}

Pale yellow solid, (167.5 mg) 85\% yield, mp: $156-158{ }^{\circ} \mathrm{C} .{ }^{1} \mathrm{H}$ NMR $\left(500 \mathrm{MHz}, \mathrm{CDCl}_{3}\right) \delta 7.96-$ $7.86(\mathrm{~m}, 2 \mathrm{H}), 7.76(\mathrm{~d}, J=7.9 \mathrm{~Hz}, 2 \mathrm{H}), 7.71-7.64(\mathrm{~m}, 2 \mathrm{H}), 7.36(\mathrm{~d}, J=7.9 \mathrm{~Hz}, 2 \mathrm{H}), 4.69$ (s, 2H), 2.48 (s, 3H). Known compound. ${ }^{4}$<smiles>O=C(CS(=O)(=O)Cl)c1ccc(I)cc1</smiles>

\section{1-p-Tolyl-2-tosylethanone (3i)}


Colorless solid, (117.5 mg) 79\% yield, mp: $103-105{ }^{\circ} \mathrm{C} .{ }^{1} \mathrm{H}$ NMR $\left(500 \mathrm{MHz}, \mathrm{CDCl}_{3}\right) \delta 7.87(\mathrm{~d}, J$ $=7.5 \mathrm{~Hz}, 2 \mathrm{H}), 7.78(\mathrm{~d}, J=7.5 \mathrm{~Hz}, 2 \mathrm{H}), 7.36(\mathrm{~d}, J=7.9 \mathrm{~Hz}, 2 \mathrm{H}), 7.30(\mathrm{~d}, J=8.8 \mathrm{~Hz}, 21 \mathrm{H}), 4.71$ (s, 2H), 2.47 (s, 3H), 2.45 (s, 3H). Known compound. ${ }^{3}$<smiles>Cc1ccc(C(=O)CS(=O)(=O)[O-])cc1</smiles>

\section{1-(Naphthalen-2-yl)-2-tosylethanone (3j)}

Colorless solid, (132.5 mg) 82\% yield, mp: $144-147{ }^{\circ} \mathrm{C} .{ }^{1} \mathrm{H}$ NMR $\left(500 \mathrm{MHz}, \mathrm{CDCl}_{3}\right) \delta 8.48$ (s, 1H), $8.04-7.96$ (m, 2H), $7.95-7.88(\mathrm{~m}, 2 \mathrm{H}), 7.80$ (d, $J=7.8 \mathrm{~Hz}, 2 \mathrm{H}), 7.67$ (t, $J=7.5 \mathrm{~Hz}, 1 \mathrm{H})$, $7.62(\mathrm{~d}, J=7.5 \mathrm{~Hz}, 1 \mathrm{H}), 7.35$ (d, $J=7.9 \mathrm{~Hz}, 2 \mathrm{H}), 4.87$ (s, 2H), 2.44 (s, 3H). Known compound. ${ }^{3}$<smiles>O=C(CS(=O)(=O)Cl)c1ccc2ccccc2c1</smiles>

\section{1-Phenyl-2-(phenylsulfonyl)ethanone (3I)}

Colorless solid, (102.5 mg) 80\% yield, mp: 93-95 ${ }^{\circ} \mathrm{C} .{ }^{1} \mathrm{H}$ NMR (500 MHz, $\left.\mathrm{CDCl}_{3}\right) \delta 7.97$ (d, J= $7.7 \mathrm{~Hz}, 2 \mathrm{H}), 7.93(\mathrm{~d}, J=7.8 \mathrm{~Hz}, 2 \mathrm{H}), 7.70(\mathrm{t}, J=7.6 \mathrm{~Hz}, 1 \mathrm{H}), 7.64(\mathrm{~d}, J=7.5 \mathrm{~Hz}, 1 \mathrm{H}), 7.58(\mathrm{t}, J$ $=7.7 \mathrm{~Hz}, 2 \mathrm{H}), 7.51(\mathrm{t}, J=7.7 \mathrm{~Hz}, 2 \mathrm{H}), 4.76(\mathrm{~s}, 2 \mathrm{H})$. Known compound. ${ }^{1}$<smiles>O=C(CS(=O)(=O)c1ccccc1)c1ccccc1</smiles>

\section{1-(4-Bromophenyl)-2-(phenylsufonyl)ethanone (3m)}

Pale yellow solid, (137.5 mg) 84\% yield, mp: $136-138{ }^{\circ} \mathrm{C} .{ }^{1} \mathrm{H}$ NMR $\left(500 \mathrm{MHz}, \mathrm{CDCl}_{3}\right) \delta 7.93-$ $7.82(\mathrm{~m}, 2 \mathrm{H}), 7.80-7.74(\mathrm{~m}, 2 \mathrm{H}), 7.66(\mathrm{dd}, J=8.7,2.3 \mathrm{~Hz}, 2 \mathrm{H}), 7.51-7.34(\mathrm{~m}, 2 \mathrm{H}), 4.69(\mathrm{~s}$, $2 \mathrm{H}), 2.48$ (s, 3H). Known compound. ${ }^{1}$<smiles>O=C(CS(=O)(=O)c1ccccc1)c1ccc(Br)cc1</smiles> 


\section{1-(4-Iodophenyl)-2-(phenylsulfonyl)ethanone (3n)}

Pale yellow solid, (157.5 mg) 82\% yield, mp: $149-151{ }^{\circ} \mathrm{C} .{ }^{1} \mathrm{H} \mathrm{NMR}\left(500 \mathrm{MHz}, \mathrm{CDCl}_{3}\right) \delta 7.89$ (s, 4H), $7.70(\mathrm{~m}, 3 \mathrm{H}), 7.59$ (m, 2H), 4.70 (s, 2H). Known compound. ${ }^{4}$<smiles>O=C(CS(=O)(=O)c1ccccc1)c1ccc(I)cc1</smiles>

\section{2-(Phenylsulfonyl)-1-p-tolylethanone (3o)}

Colorless solid, (100 mg) 74\% yield, mp: $120-123{ }^{\circ} \mathrm{C} .{ }^{1} \mathrm{H}$ NMR $\left(500 \mathrm{MHz}, \mathrm{CDCl}_{3}\right) \delta 7.91(\mathrm{~d}, J=$ $7.8 \mathrm{~Hz}, 2 \mathrm{H}), 7.86(\mathrm{~d}, J=7.9 \mathrm{~Hz}, 2 \mathrm{H}), 7.68(\mathrm{t}, J=7.5 \mathrm{~Hz}, 1 \mathrm{H}), 7.57(\mathrm{t}, J=7.8 \mathrm{~Hz}, 2 \mathrm{H}), 7.30(\mathrm{~d}, J$ $=8.4 \mathrm{~Hz}, 2 \mathrm{H}), 4.73(\mathrm{~s}, 2 \mathrm{H}), 2.44(\mathrm{~s}, 3 \mathrm{H})$. Known compound. ${ }^{3}$<smiles>Cc1ccc(C(=O)CS(=O)(=O)c2ccccc2)cc1</smiles>

\section{1-(Naphthalen-2-yl)-2-(phenylsulfonyl)ethanone (3p)}

Colorless solid, (120 mg) 78\% yield, mp: $143-146{ }^{\circ} \mathrm{C} .{ }^{1} \mathrm{H}$ NMR (500 MHz, $\left.\mathrm{CDCl}_{3}\right) \delta 8.50$ (s, 1H), $7.99(\mathrm{t}, J=8.2 \mathrm{~Hz}, 2 \mathrm{H}), 7.96-7.89(\mathrm{~m}, 4 \mathrm{H}), 7.71-7.65(\mathrm{~m}, 2 \mathrm{H}), 7.64-7.54(\mathrm{~m}, 3 \mathrm{H}), 4.89$ (s, 2H). Known compound. ${ }^{3}$<smiles>O=C(CS(=O)(=O)c1ccccc1)c1ccc2ccccc2c1</smiles> 


\section{IV- Copies of ${ }^{1} \mathrm{H}$ NMR, Mass, and IR}

1-Phenyl-2-tosylethanone (3a): ${ }^{1} \mathrm{H}$ NMR, Mass and IR<smiles>O=C(CS(=O)(=O)[GeH3])c1ccccc1</smiles>
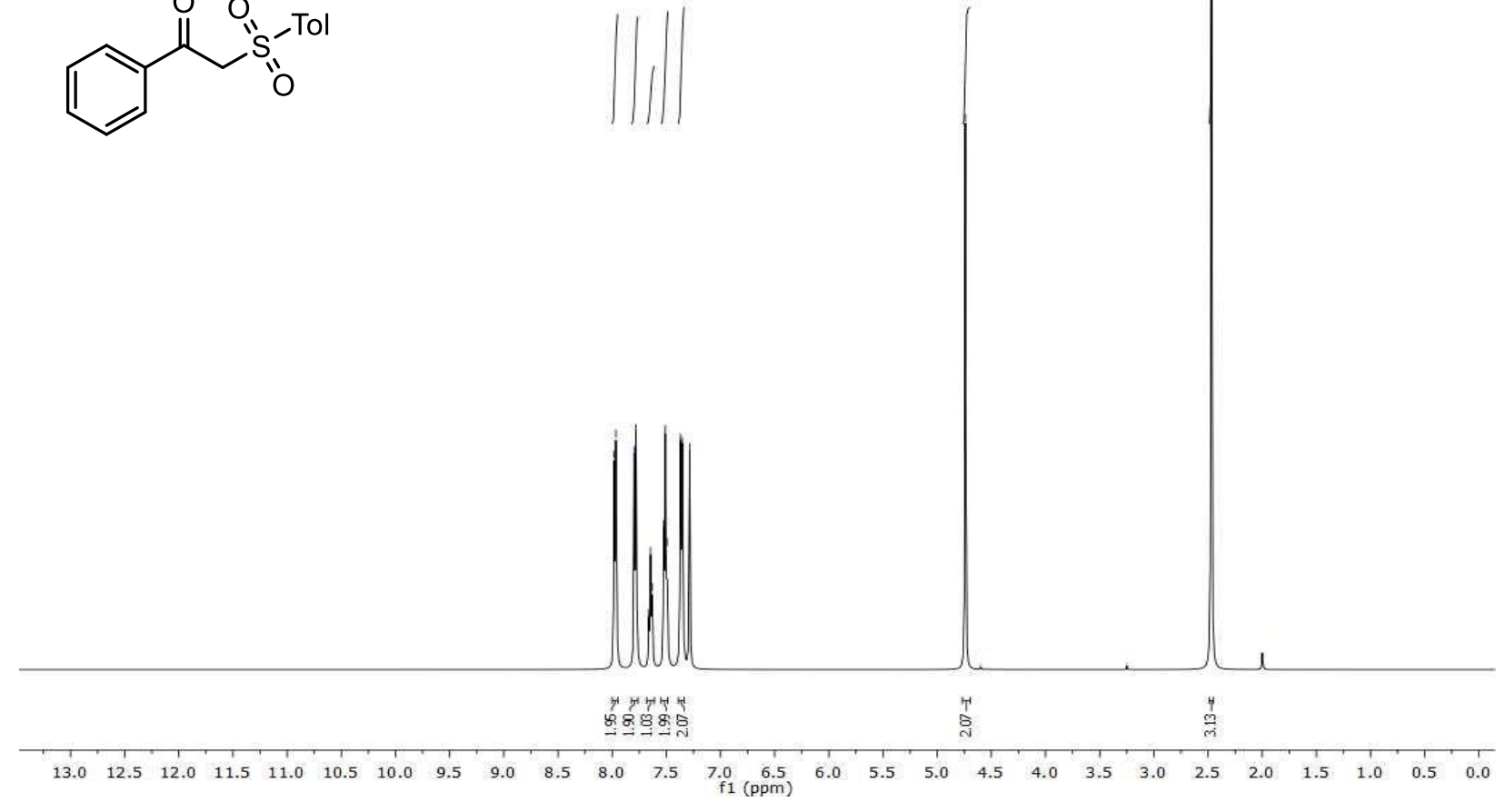

Acquired : 2 Jun 2019 12:04 using AcqMethod default.597x.m

Instrument: Direct Probe

Sample Name:

Misc Info:

Vial Number: 1

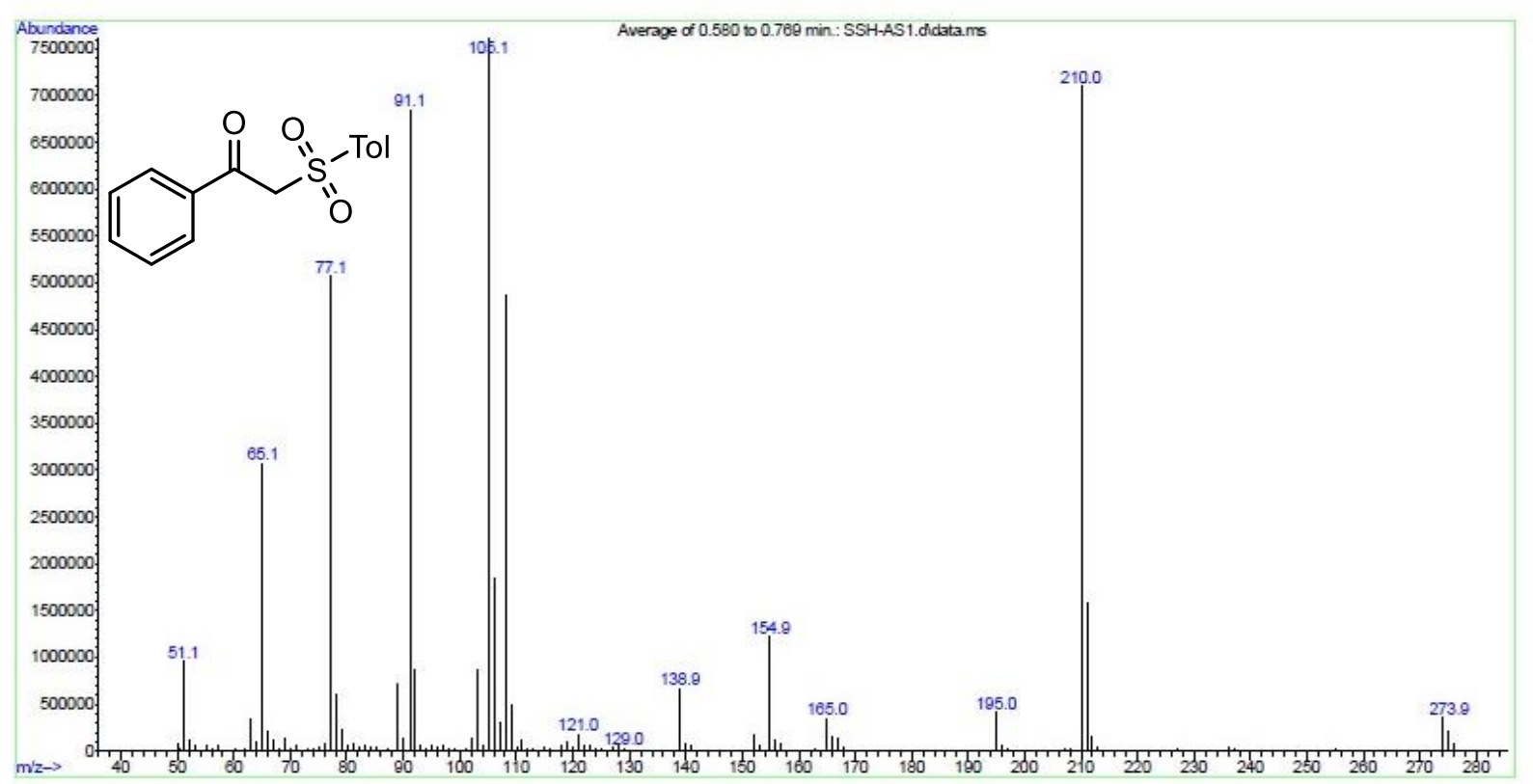




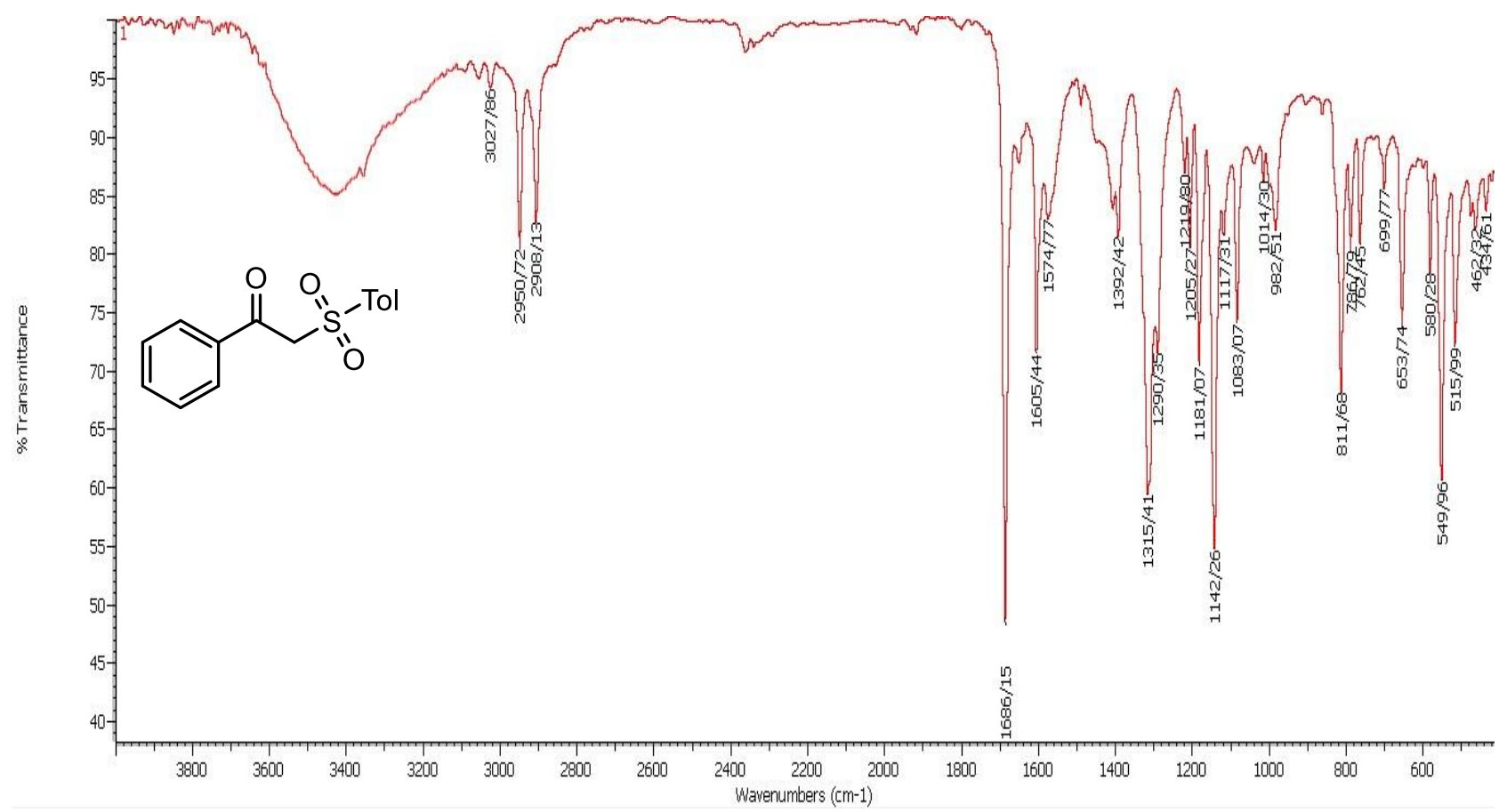

1-(o-Tolyl)-2-tosylethan-1-one (3b): ${ }^{1} \mathrm{H}$ NMR<smiles>Cc1ccccc1C(=O)CS(=O)(=O)[O-]</smiles>

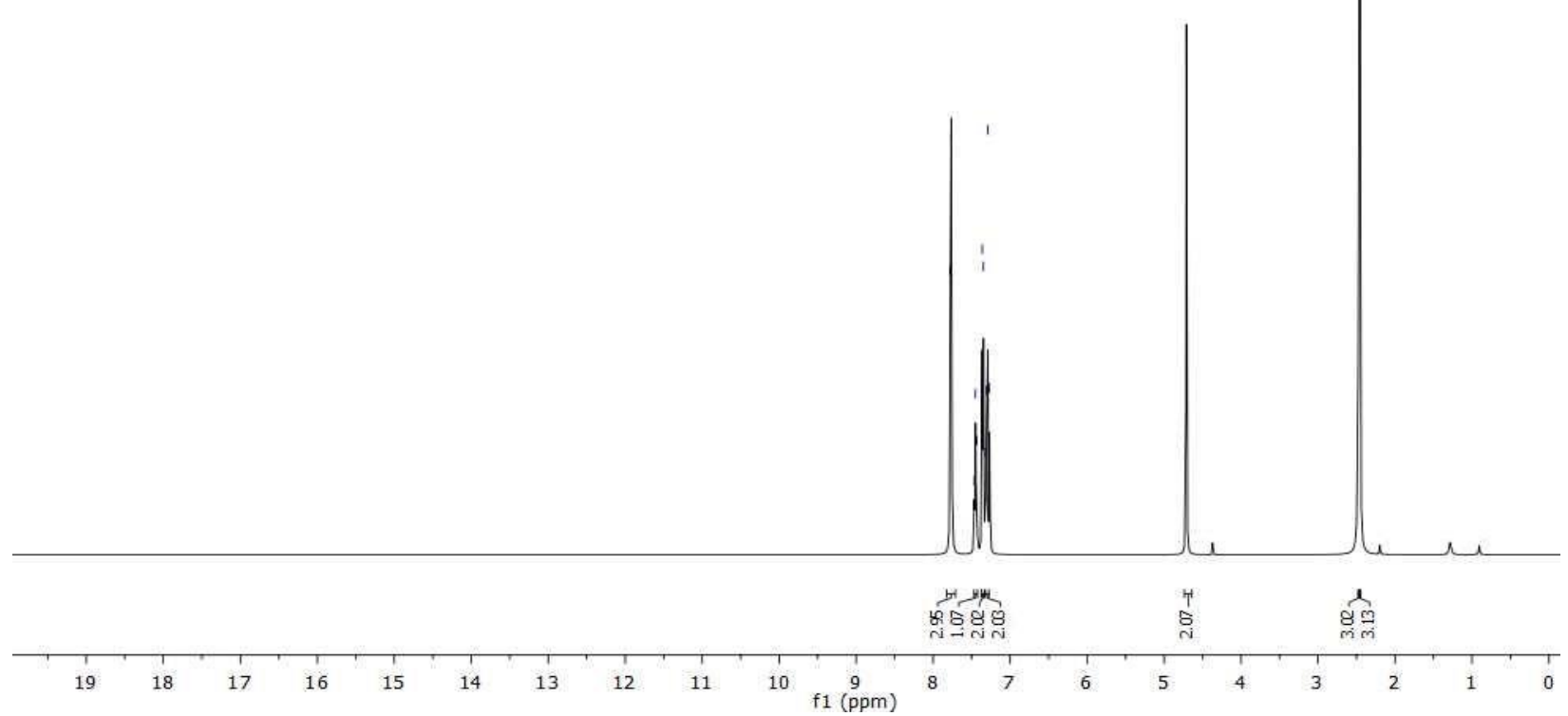




\section{1-(2-Chlorophenyl)-2-tosylethanone (3c)}

Guest.5991.fid

Shabanzade SSHAS14 1HNMR in CDCl3 at 298k 1398/05/06

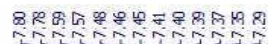

क्ष<smiles>O=C(CS(=O)(=O)[AlH2])c1ccccc1Cl</smiles>
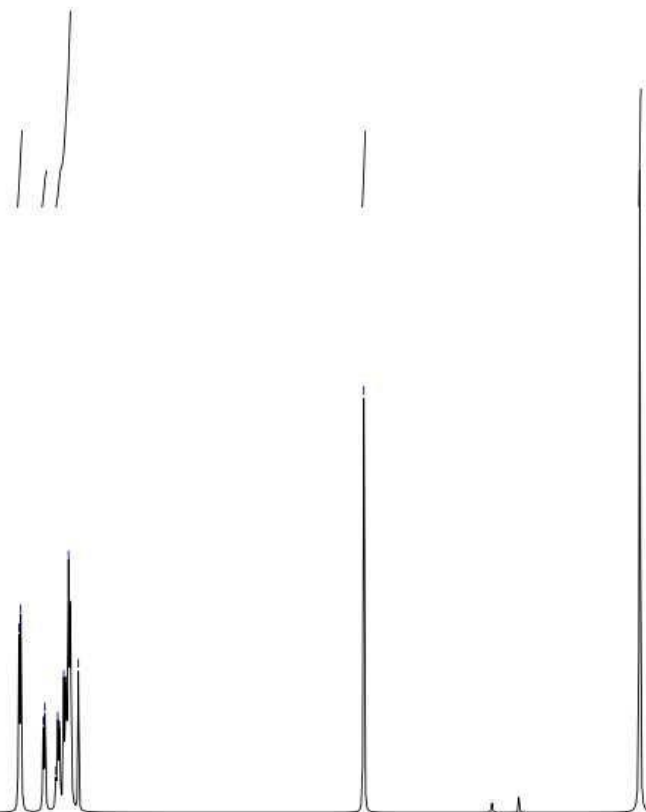

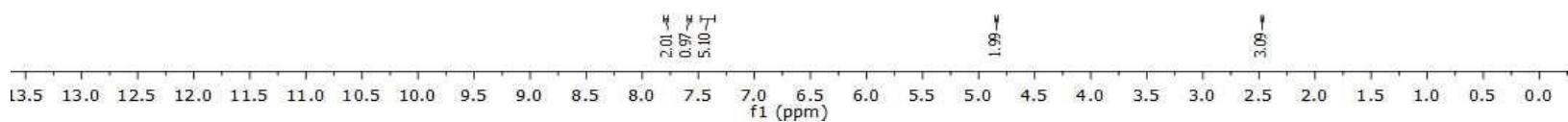

\section{1-(2-Methoxyphenyl)-2-tosylethanone (3d)}

Guest.5991.fid<smiles>COc1ccccc1C(=O)CS(=O)(=O)[GeH3]</smiles>

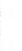




\section{1-(4-Fluorophenyl)-2-tosylethanone (3e)}

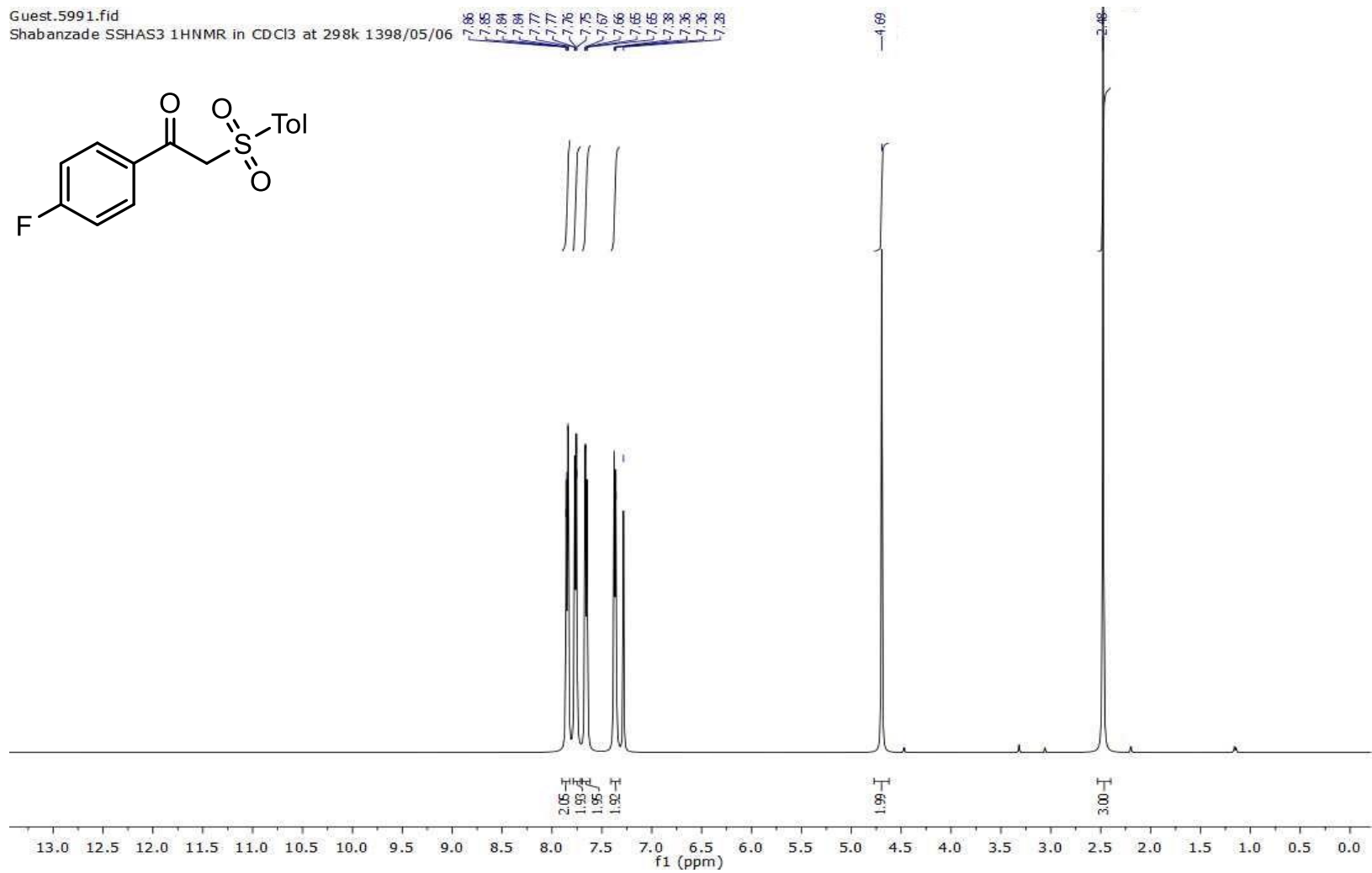

\section{1-(4-Bromophenyl)-2-tosylethanone (3f)}

Guest.5991.fid

Shabanzade SSHAS15 1 HNMR in CDCl3 at 298k 1398/05/06<smiles>O=C(CS(=O)(=O)[AlH2])c1ccc(Br)cc1</smiles>

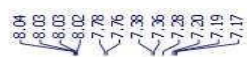

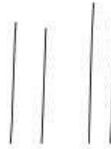

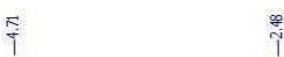

1

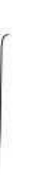

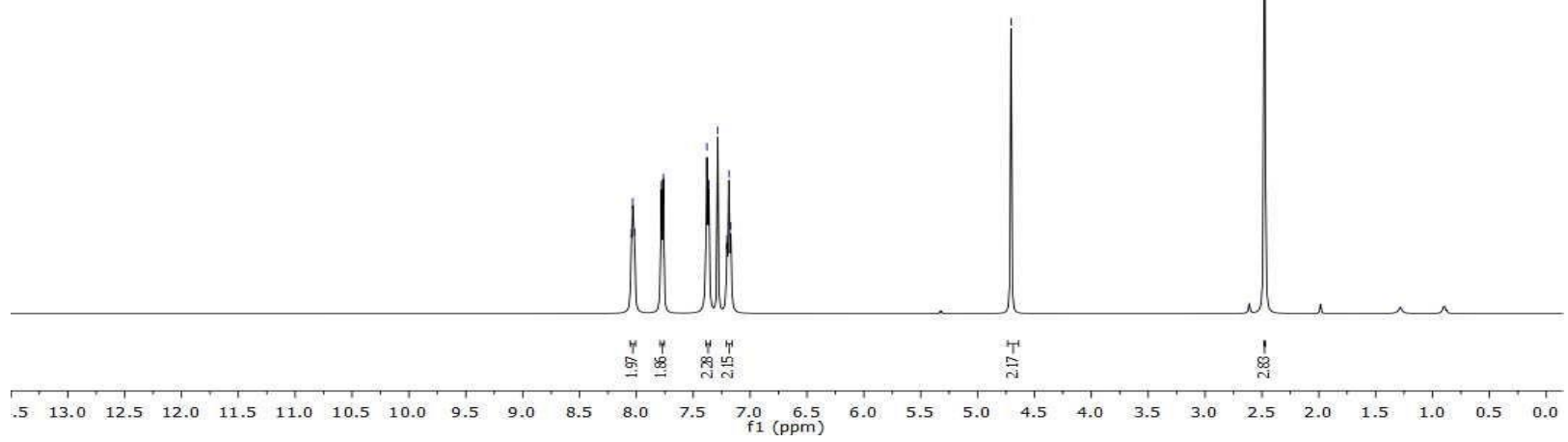




\section{1-(4-Iodophenyl)-2-tosylethanone (3g)}

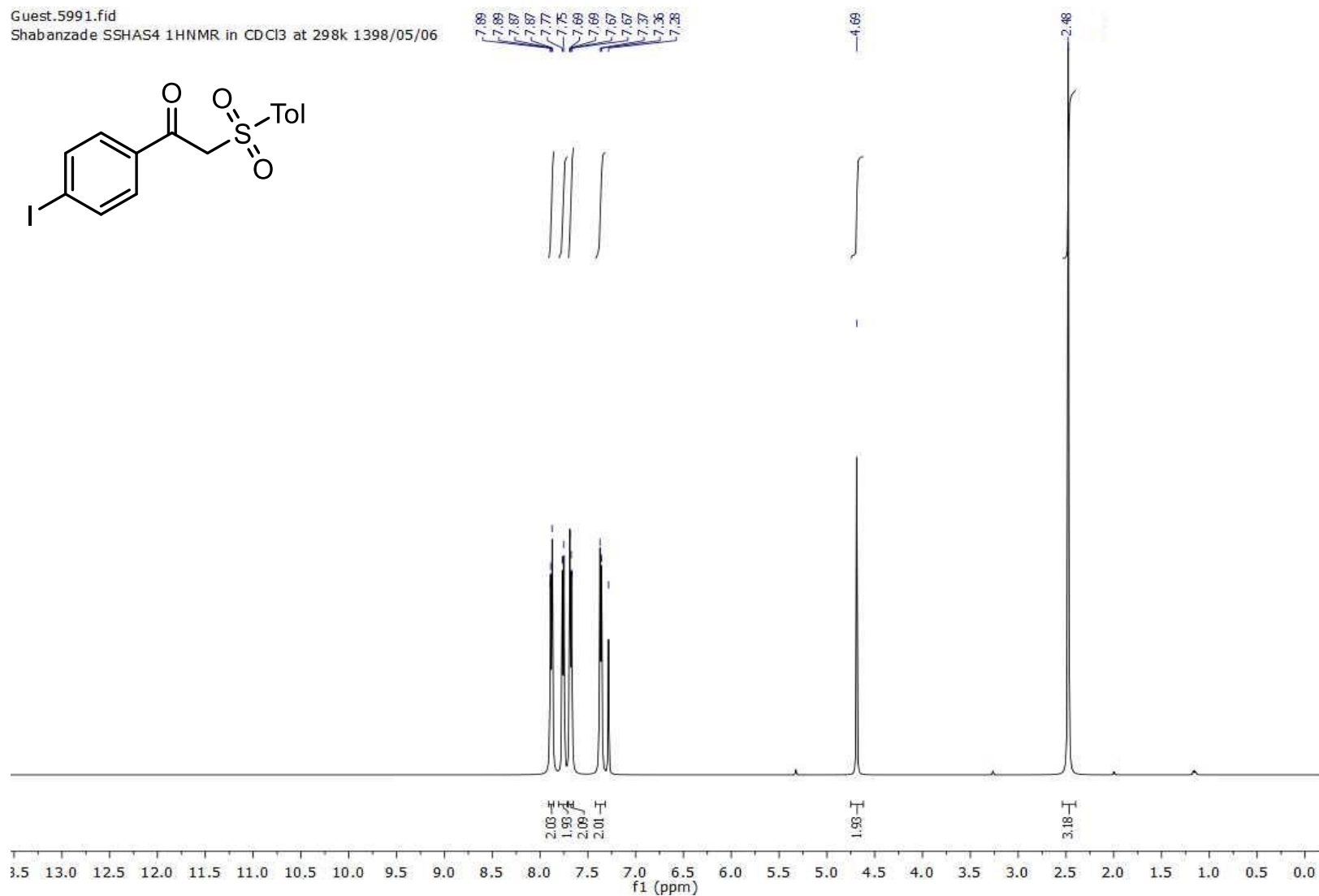

\section{1-p-Tolyl-2-tosylethanone (3i)}

Guest.5991.fid

Shabanzade SSHAS6 1 HNMR in $\mathrm{CDCl} 3$ at 298k 1398/05/06

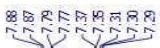

$\stackrel{1}{i}$<smiles>Cc1ccc(C(=O)CS(=O)(=O)[GeH2])cc1</smiles><smiles>c1ccccc1</smiles>
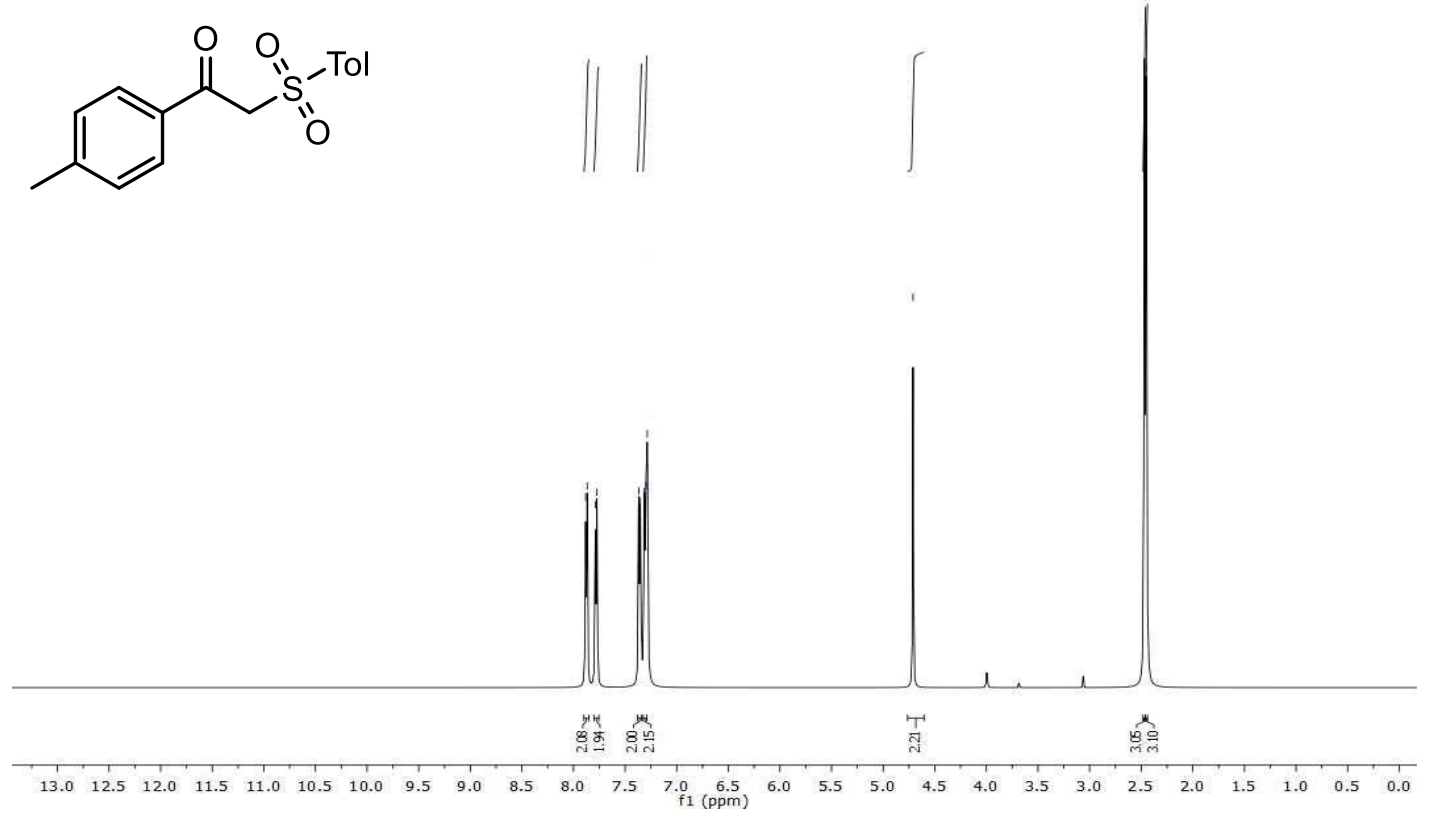
1-(Naphthalen-2-yl)-2-tosylethanone (3j)

Guest.5991.fid
Shabanzade SSHAS2 $1 \mathrm{HNMR}$ in $\mathrm{CDCl} 3$ at $298 \mathrm{~K} 1398 / 05 / 06$

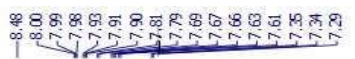

$\stackrel{\infty}{i}$

$\stackrel{\text { Z }}{i}$<smiles>O=C(CS(=O)(=O)[AlH2])c1ccc2ccccc2c1</smiles>
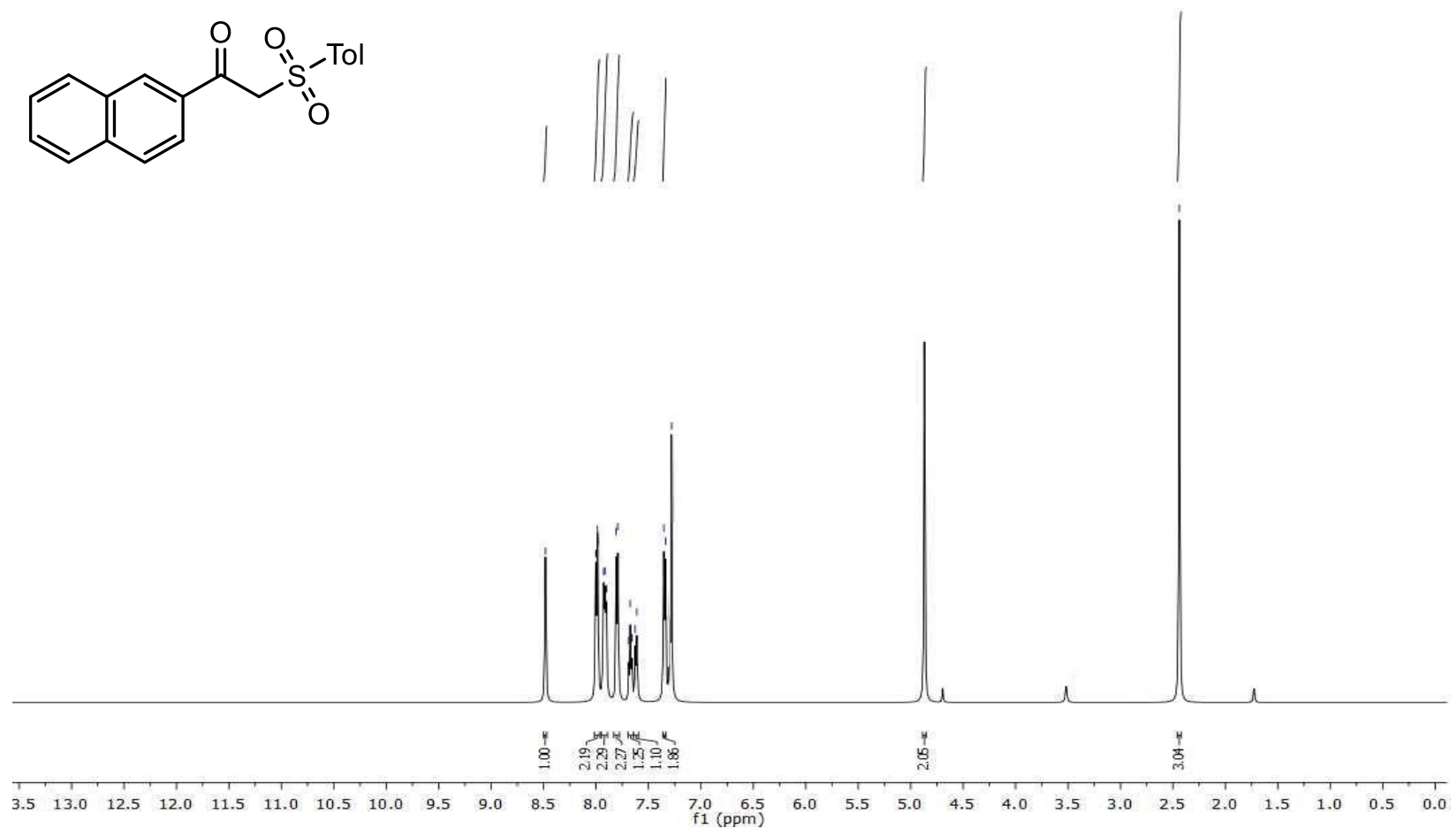

1-Phenyl-2-(phenylsulfonyl)ethanone (3l) 


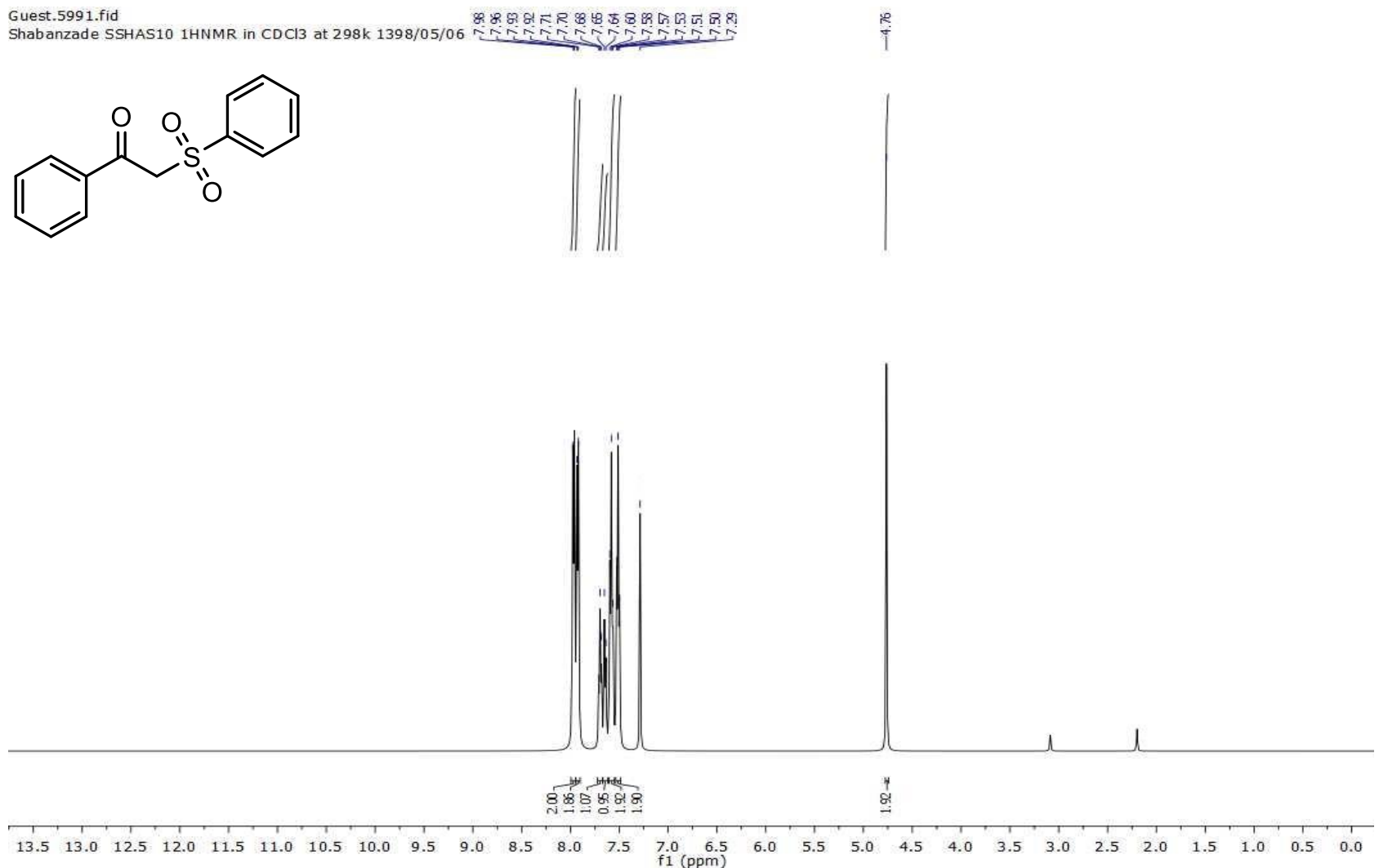

\section{1-(4-Bromophenyl)-2-(phenylsufonyl)ethanone (3m)}

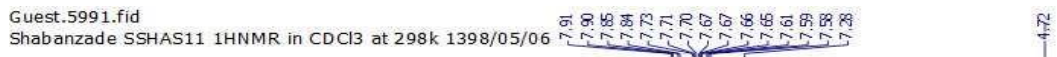<smiles>O=C(CS(=O)(=O)c1ccccc1)c1ccc(Br)cc1</smiles>
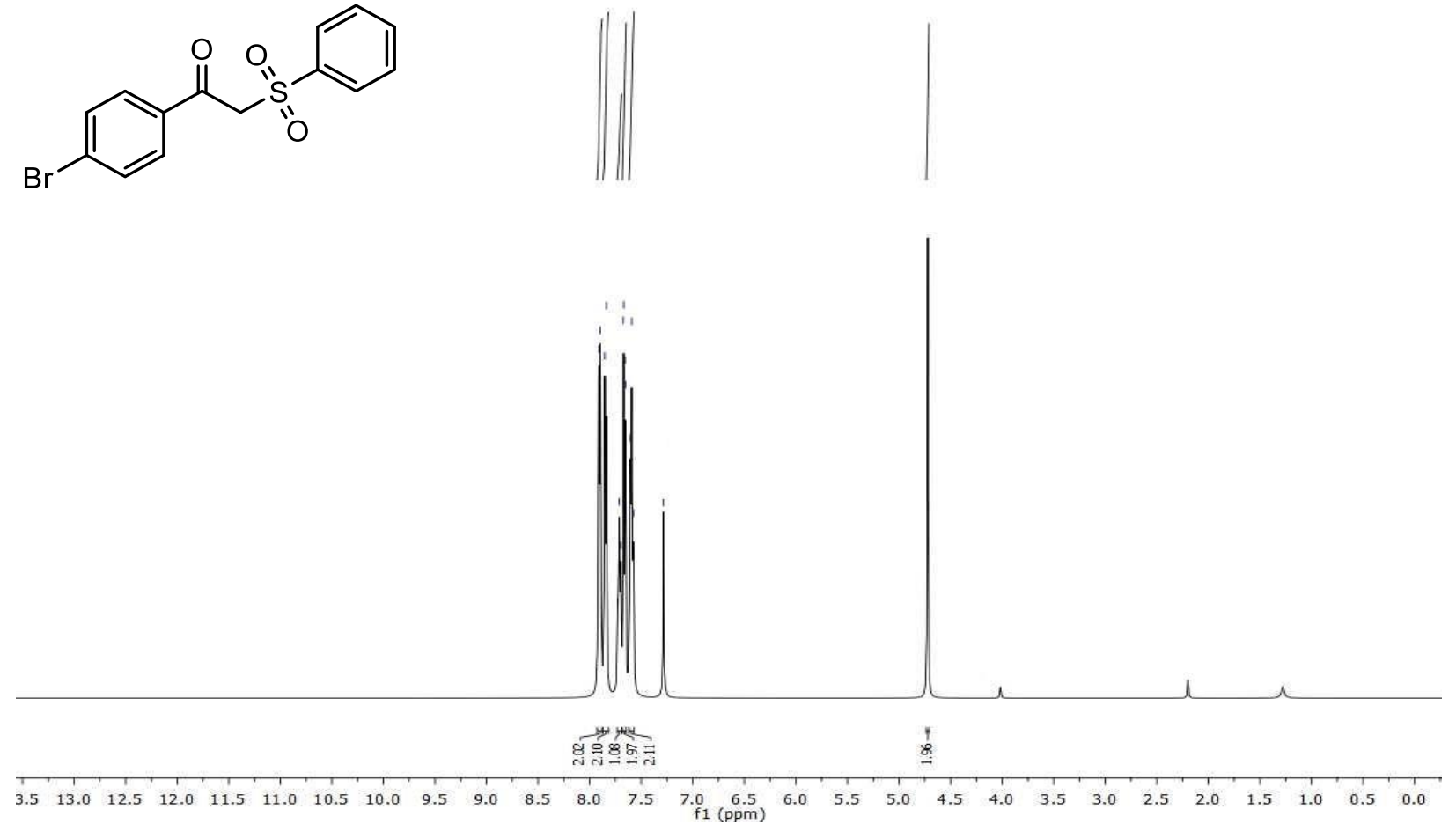


\section{1-(4-Iodophenyl)-2-(phenylsulfonyl)ethanone (3n)}

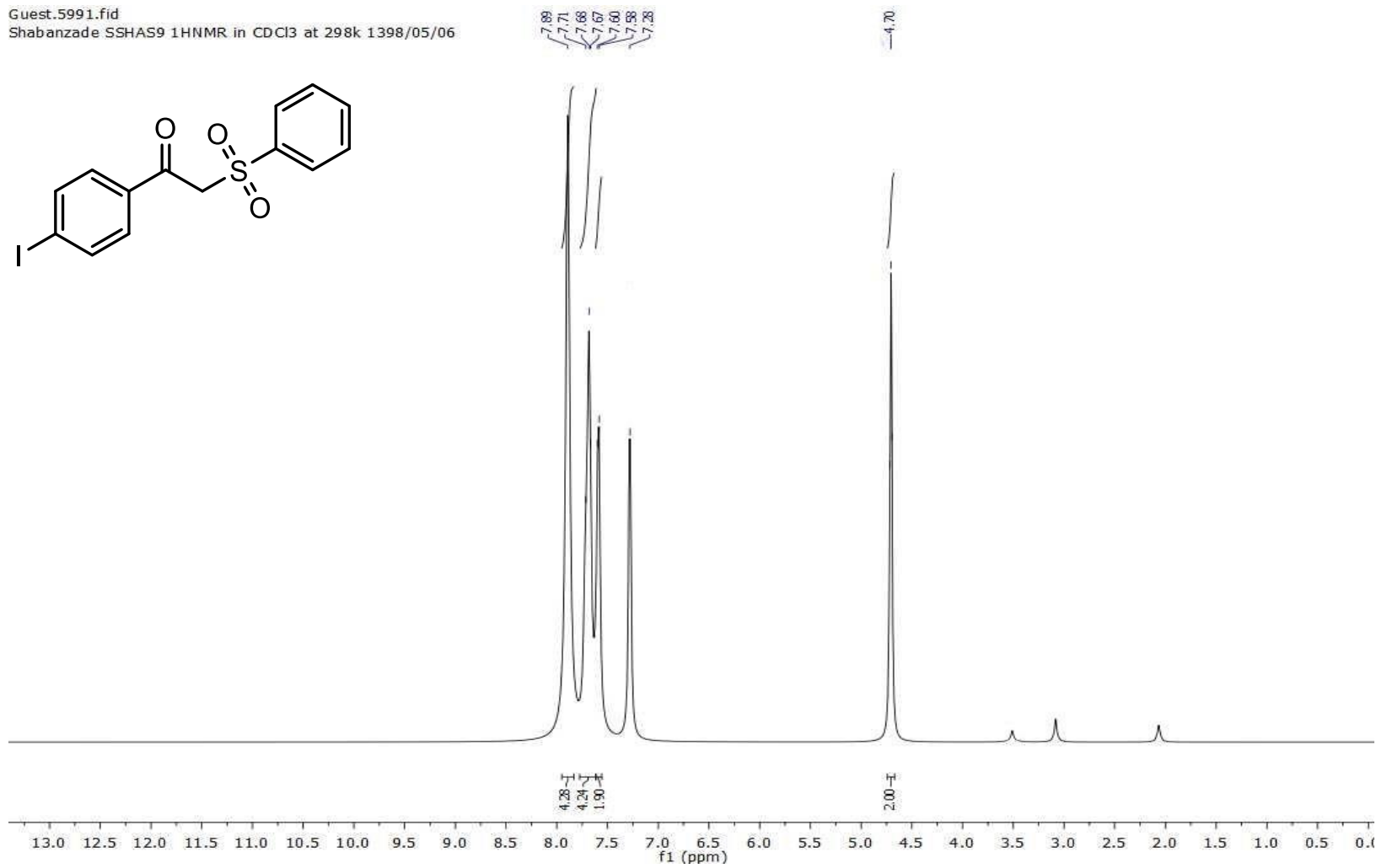

\section{2-(Phenylsulfonyl)-1-p-tolylethanone (3o)}

Guest.5991.fid

Shabanzade SSHAS12 1HNMR in CDCl3 at 298k 1398/05/06

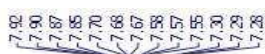<smiles>Cc1ccc(C(=O)CS(=O)(=O)c2ccccc2)cc1</smiles>

$\stackrel{k}{i}$

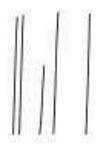

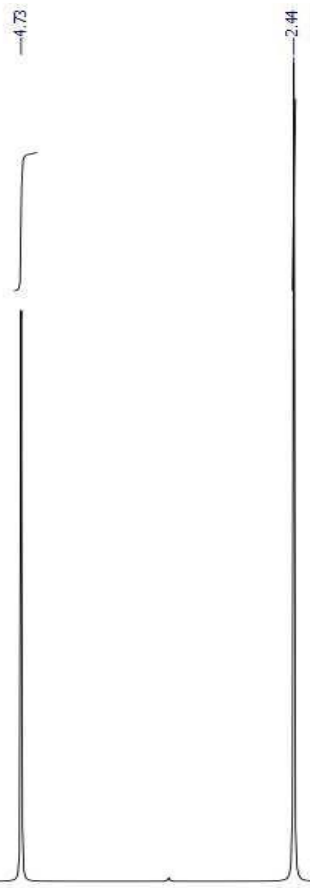

Tु

$\stackrel{f}{\circ}$

\begin{tabular}{lllllllllllllllllllllllllllllll}
\hline .5 & 13.0 & 12.5 & 12.0 & 11.5 & 11.0 & 10.5 & 10.0 & 9.5 & 9.0 & 8.5 & 8.0 & 7.5 & 7.0 & 6.5 & 6.0 & 5.5 & 5.0 & 4.5 & 4.0 & 3.5 & 3.0 & 2.5 & 2.0 & 1.5 & 1.0 & 0.5 & 0.0
\end{tabular} 


\section{1-(Naphthalen-2-yl)-2-(phenylsulfonyl)ethanone (3p)}

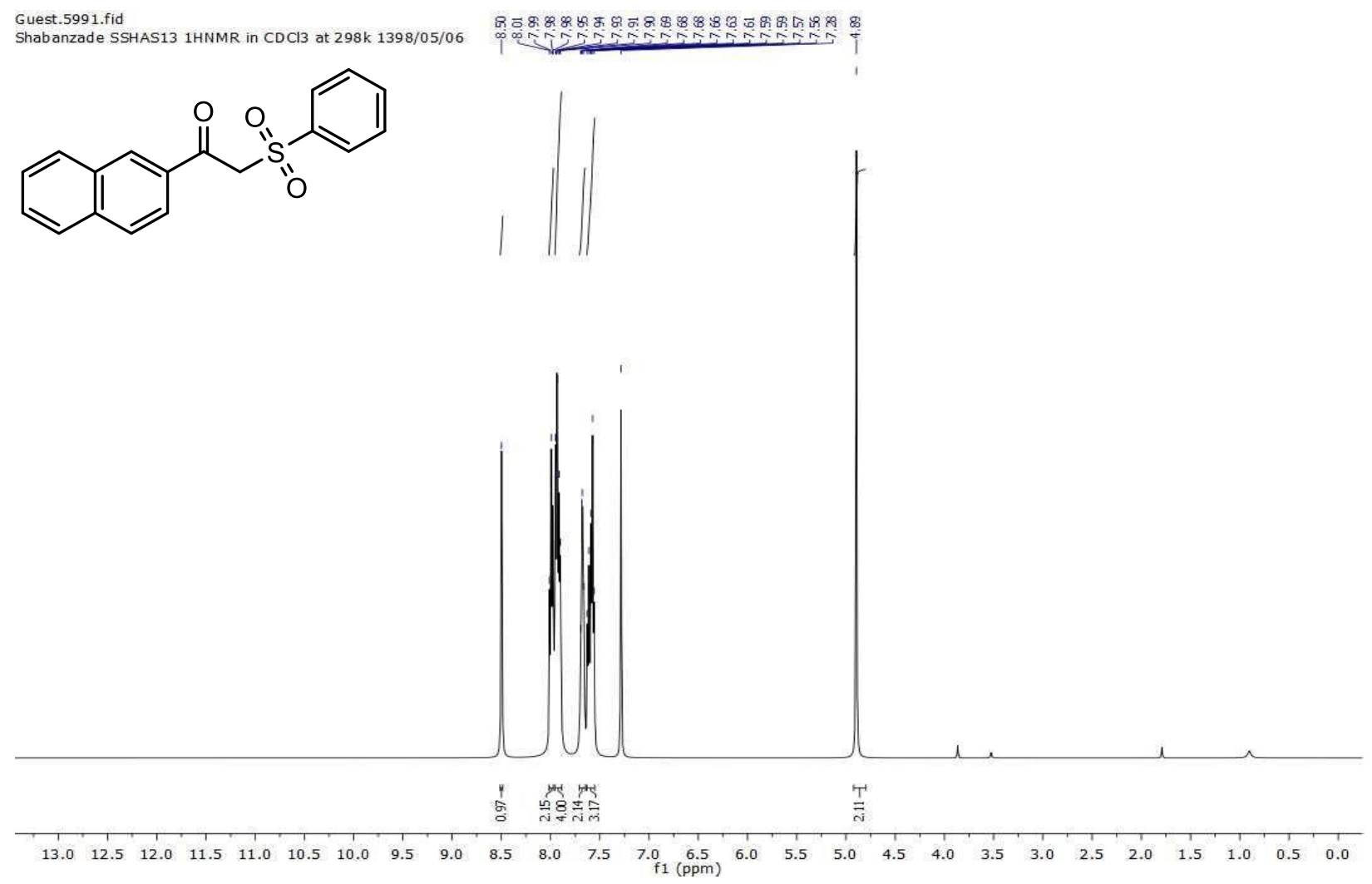

\section{V-Reference}

1. Handa, S.; Fennewald, J. C.; Lipshutz, B. H. Angew. Chem. Int. Ed. Engl. 2014, 53, 3432.

2. Lin, B.; Kuang, J.; Chen, J.; Hua, Z.; Khakyzadeh, V.; Xia, Y. Org. Chem. Front. 2019, $6,2647$.

3. Yang, D.; Huang, B.; Wei, W.; Li, J.; Lin, G.; Liu, Y.; Ding, J;. Sun, P.; Wang, H. Green Chem. 2016, 18, 5630.

4. Rawat, V. S.; Reddy, P. L.; Sreedhar, B. RSC Adv. 2014, 4, 5165.

5. (a) Kang, J. C.; Tu, Y. Q.; Dong, J. W.; Chen, C.; Zhou, J.; Ding, T. M.; Zai, J. T.; Chen, Z. M. Org. Lett. 2019, 21, 2536. (b) Jiang, Y. Y.; Dou, G. Y.; Xu, K.; Zeng, C. C. Org. Chem. Front. 2018, 5, 2573. (c) Sheykhan, M.; Khani, S.; Abbasnia, M.; Shaabanzadeh, S.; Joafshan, M. Green Chem. 2017, 19, 5940. 